VOL. 17 (1977), 391-399.

\title{
Multiplicative functionals and a class of topological algebras
}

\section{Gerard A. Joseph}

\begin{abstract}
Every multiplicative linear functional on a pseudocomplete locally convex algebra satisfying the "sequential" property of Husain and $\mathrm{Ng}$ is bounded (a topological algebra is called "sequential" if every null sequence contains an element whose powers converge to zero). Characterizations of such algebras are given, with some examples.
\end{abstract}

\section{Introduction}

This paper is essentially an expansion of that of Husain and $\mathrm{Ng}$ [5], in which the concept of a sequential topological algebra is introduced. Their main result ([5], p. 500) asserts that on a sequentially complete, sequential locally convex algebra, each multiplicative linear functional is bounded (maps bounded sets to bounded sets). Modulo the sequential condition, then, this gives an affirmative answer to Michael's original question ([7], p. 50) without requiring the algebra to be either commutative or locally multiplicatively-convex.

We give a simplified proof (Theorem 2.1) of Husain and $\mathrm{Ng}$ 's result, using some ideas of Allan [1]. At the same time we can relax the completeness condition required, the appropriate condition being Allan's pseudocompleteness.

In $\$ 3$ we give some equivalent formulations of the sequential property, motivated by Proposition 2 of [5], which implies that a sequentially

Received 29 June 1977. The author gratefully acknowledges the support of a Rotary Fellowship, and the helpful comments of E.A. Michael and R.T. Moore. 
complete locally multiplicatively-convex $Q$-algebra is sequential; in a Q-algebra, however, modular maximal ideals are closed $([8], p .78)$, in particular those of codimension 1 , which is tantamount to the actual continuity of every multiplicative linear functional. In $\$ 4$ we exhibit some sequential algebras which are not $Q$-algebras.

A summary of the basic concepts used in this paper appears in $\$ 1$.

\section{Preliminaries}

A topological algebra is an algebra (assumed complex) with a Hausdorf linear topology in which vector multiplication is separately continuous; if the topology is locally convex, the algebra is called a zocally convex algebra; if, further, there is a neighborhood base $u$ at 0 such that each $U \in U$ is convex, balanced $(\lambda U \subseteq U$ if $|\lambda| \leq 1)$, and a semigroup $(x y \in U$ for all $x, y \in U)$, the algebra is called zocally multiplicatively-convex. Every locally multiplicatively-convex algebra has jointly continuous multiplication. A complete, metrizable, locally multiplicatively-convex algebra is called a Frechet algebra.

Let $A$ be an algebra. For $a, b \in A$ we define $a \circ b=a+b+a b$. An element $a \in A$ is quasi-reguzar if there exists $b \in A$ (its quasiinverse) satisfying $a \circ b=b \circ a=0$. If $A$ has an identity 1 , then $a$ is quasi-regular if and only if $a+1$ is invertible. A Q-algebra is a topological algebra in which the set of quasi-regular elements is open.

The spectrum of an element $a$ of an algebra is the set of scalars

$$
\sigma(\alpha)=\left\{\lambda:-\lambda^{-1} a \text { is not quasi-regular }\right\} \cup \sigma_{1}(a),
$$

where $\sigma_{1}(a)=\emptyset$ if $a$ i.s invertible, $\sigma_{1}(a)=\{0\}$ otherwise. The spectral radius of $a$ is the number $\rho(a)=\sup \{|\lambda|: \lambda \in \sigma(\alpha)\}$, where $\rho(a)=\infty$ is admitted.

\section{Main result}

The following notions, due to $A / I$ an $[1]$, are pertinent to our main result. A denotes a locally convex algebra.

The radius of boundedness of an element $a \in A$ is the number 


$$
B(a)=\inf \left\{r>0: r^{-1} a \text { generates a bounded semigroup }\right\} \text {, }
$$

where inf $\varnothing=\infty$; (elements for which $B(a)<\infty$ are called bounded elements). The following properties ([1], p. 405) are easy to verify:

(1) $\beta(\lambda a)=|\lambda| \beta(\alpha), \lambda$ scalar (here $0 . \infty=0)$,

(2) $\infty>|\lambda|>B(a)$ implies $\left(\lambda^{-1} a\right)^{n} \rightarrow 0$ as $n \rightarrow \infty$.

Denote by $A$ the class of all closed, convex, balanced, bounded semigroups in $A$. Given $B \in A$, the linear span $A_{B}$ of $B$ is a subalgebra of $A$. For $a \in A_{B}$, define $\|a\|_{B}=\inf \left\{r>0: r^{-1} a \in B\right\}$. Then $\left(A_{B},\|\cdot\|_{B}\right)$ is a normed algebra with unit ball $B . A$ is said to be pseudocomplete in case $\left(A_{B},\|\cdot\|_{B}\right)$ is complete for all $B \in A$. We remark that if $a \in A$ generates a bounded semigroup, then $a \in B$ for some $B \in A$; for example, take $B$ to be the closed convex balanced hull of $\left\{a^{n}: n=1,2, \ldots\right\} ;($ see [7], Lemmas 1.3 and 1.4$)$.

A sequentially complete algebra is pseudocomplete ([1], p. 40I); a normed algebra is pseudocomplete if and only if it is complete.

We recall the definition of a sequential algebra.

DEFINITION. A topological algebra is sequential if for every sequence $\left\{x_{n}\right\}$ converging to 0 , there exists $n_{0}$ such that $x_{n_{0}}^{k} \rightarrow 0$ as $k \rightarrow \infty$ (in which case $x_{n}^{k} \rightarrow 0$ for eventually all $n$ ).

A normed algebra is clearly sequential; further, each element $a$ of a sequential locally convex algebra is bounded in the sense defined above (consider the sequence $\left\{n^{-1} a\right\}$ ).

THEOREM 2.1. Let $A$ be a pseudocomplete, sequential locally convex algebra. Then each multiplicative linear functional $f$ on $A$ is bounded.

Proof. We first observe that $|f(a)| \leq B(a)$ for all $a € A$; if $r>0$ such that $r^{-1} a$ generates a bounded semigroup, then $r^{-1} a \in B$ for some $B \in A$, and $\left(A_{B},\|\cdot\|_{B}\right)$ is a Banach algebra; considering the. restriction of $f$ to $A_{B}$, we have $|f(a)| \leq\|a\|_{B} \leq r$; we deduce that 
$|f(a)| \leq \beta(a)$. Suppose now that $f$ is not bounded. Then there is a bounded sequence $\left\{x_{n}\right\}$ in $A$ such that $\left|f\left(x_{n}\right)\right|>n$; since $n^{-1} x_{n} \rightarrow 0$, for some $n_{0}$ we have $\left(n_{0}^{-1} x_{n_{0}}\right)^{k}+0$ as $k \rightarrow \infty$, so $\beta\left(x_{n_{0}}\right) \leq n_{0}<\left|f\left(x_{n_{0}}\right)\right|$, a contradiction.

\section{The sequential property}

It is clear, as observed in [5], that an algebra in which there is a neighborhood $U$ of 0 such that $x^{n} \rightarrow 0$ for all $x \in U$ is sequential. We shall call such an algebra strongly sequential. In the following three propositions, $A$ denotes a topological algebra.

PROPOSITION 3.1. Let $A$ be metrizable. Then $A$ is sequential if and only if $A$ is strongly sequential.

Proof. Suppose $A$ is sequential but not strongly sequential. Let $\left\{U_{n}: n \geq 1\right\}$ be a decreasing neighborhood base at 0 . Then for each $n$ there is some $x_{n} \in U_{n}$ such that $\left\{x_{n}^{k}: k \geq 1\right\}$ does not converge to 0 . Since $x_{n} \rightarrow 0$, this contradicts the assumption that $A$ is sequential.

PROPOSITION 3.2. A is sequential if and on ly if for each sequence $\left\{x_{n}\right\}$ converging to 0 , some $x_{n_{0}}$ generates a bounded semigroup (in which case so does $x_{n}$ for eventually all $n$ ).

Proof. The 'only if' part is trivial; for the converse, let $x_{n} \rightarrow 0$, and apply the hypothesis to the sequence $\left\{2 x_{n}\right\}$.

The next characterization is proved exactly as Proposition 3.1.

PROPOSITION 3.3. Let $A$ be metrizable. Then $A$ is sequential if and only if there is a neighborhood of 0 each element of which generates a bounded semigroup.

We turn to the relation between Q-algebras and sequential algebras. Husain and $\mathrm{Ng}$ ([5], Proposition 2) show that a sequentially complete locally multiplicatively-convex algebra is a Q-algebra if and only if it 
is strongly sequential. Combining this result with Proposition 13.5 of [7], p. 55, and our Proposition 3.1, we obtain:

PROPOSITION 3.4. The folzowing are equivazent for a Fréchet algebra $A$ :

(1) A is a Q-algebra;

(2) A is sequential;

(3) $\{x \in A: \rho(x) \leq 1\}$ is a neighborhood of 0 .

It follows that $C^{\infty}[0,1]$ is sequential but that $C(\mathbb{R})$, with topology of compact convergence, is not.

We have observed that each element of a sequential locally convex algebra is bounded in the sense of $\$ 2$. Under certain conditions the reverse implication also holds, as shown by the following proposition. A locally convex algebra will be called m-barrelled if every closed, convex, balanced, absorbing semigroup is a neighborhood of 0 .

PROPOSITION 3.5. Let $A$ be a complete, conmutative, m-barrelled, locally multiplicatively-convex algebra (for excomple a commutative Fréchet algebra). Then the following are equivalent:

(1) $A$ is a Q-algebra;

(2) $A$ is sequential;

(3) $\{x \in A: \rho(x) \leq 1\}$ is a neighborhood of 0 ,

(4) $\rho(x)<\infty$ for alz $x \in A$;

(5) $\beta(x)<\infty$ for alz $x \in A$;

(6) the space $M(A)$ of continuous multiplicative linear functionals on $A$ is compact in the weak topology $\sigma\left(A^{*}, A\right)$.

Proof. The equivalence of $(1),(3),(4)$, and $(6)$ is the content of Proposition 13.5 and Theorem 13.6 of [7], pp. 55, 56. The equivalence of (4) and (5) follows from (4.2) of [1], p. 414. Finally, we have already noted that (1) implies (2) and that (2) implies (5).

\section{Examples}

We give three examples of non-metrizable algebras satisfying the hypotheses of Theorem 2.1, which are not Q-algebras. It will be useful to 
state here as a lemma the following simple fact.

LEMMA 4.1. Let $A$ be a topological algebra such that every Cauchy sequence in $A$ is Cauchy with respect to a finer, Banach algebra topology on $A$. Then $A$ is sequentially complete and sequential.

The first two examples are function algebras, after Examples 3.7 and 3.8 and Proposition 12.2 of Michael [7].

EXAMPLE 4.2. Let $X$ be an uncountable compact metric space; let $A=\mathcal{C}(X)$ have the topology of uniform convergence on countable, compact sets.

It is not difficult to check that a Cauchy sequence $\left\{f_{n}\right\}$ in $A$ is actually Cauchy in the supremum norm on $A$ (the contrary assumption implies the existence of a countable, compact subset of $X$ on which $\left\{f_{n}\right\}$ is not Cauchy with respect to uniform convergence). Now apply Lemma 4.1.

To see that $A$ is not a $Q$-algebra, we note that a neighborhood base at the identity is given by all sets of the form $\{f \in A:|1-f(x)| \leq \varepsilon$ for all $x \in K\}$, where $K$ is countable and compact, and $E>0$. Since $X$ is uncountable and completely regular, every such neighborhood contains a function which is identically $l$ on $K$ and varishes at some point elsewhere, and is thus non-invertible.

REMARK. Of course, every multiplicative linear functional on this algebra is actually continuous, since it is just a point evaluation corresponding to some point of $X$.

EXAMPLE 4.3. Let $X$ be a non-compact completely regular space with the property that every infinite subset contains an infinite subset with compact closure. Let $A=C(X)$ have the compact-open topology.

Observe that such a space $X$ is pseudocompact (every continuous function is bounded), so that $A$ is a Banach algebra with respect to the supremum norm. Then every Cauchy sequence in $A$ is actually Cauchy in this norm, so that Lemma 4.1 applies again. The non-compactness and complete regularity of $X$ ensure that $A$ is not a Q-algebra, by an argument similar to that in Example 4.2 .

REMARKS 1. The somewhat artificial condition imposed on $X$ is satisfied by any countably compact $k$-space (see [6], p. 230); first 
countable and locally compact spaces are $k$-spaces. A concrete example is the space of ordinals less than the first uncountable ordinal, in the order topology.

2. On this algebra the multiplicative linear functionals are precisely the point evaluations corresponding to $\beta(X)$, the Stone-Čech compactification of $X$. Michael's Proposition 12.2 ([7], p. 49) shows directly that all such functionals are bounded, and further that those corresponding to $\beta(X) \backslash X$ are not continuous.

Our third example, an operator algebra, is similar in spirit to the other two, but in contrast is neither commutative nor locally multiplicatively-convex.

EXAMPLE 4.4. Let $X$ be a nonseparable reflexive Banach space, $B(X)$ the Banach algebra of bounded linear operators on $X$. Let $A$ be any closed subalgebra of $B(X)$ containing all operators in $B(X)$ of finite rank, with the topology of uniform convergence on countable, weakly compact sets.

Suppose that $\left\{T_{n}\right\}$ is a Cauchy sequence in $A$ which is not Cauchy in the operator norm on $A$. Then there exist $\varepsilon>0$ and $x_{n} \in X$, $\left\|x_{n}\right\|=1$, such that for each $n$,

$$
\left\|T_{k_{1}}(n)^{x}{ }^{-T} k_{2}(n)^{x}\right\| \geq \varepsilon \text { for some } k_{1}(n), k_{2}(n)>n
$$

Since $X$ is reflexive, the Eberlein-Šmulian Theorem ([4], p. 430) implies that $\left\{x_{n}\right\}$ contains a weakly convergent subsequence, which with its limit adjoined is a countable, weakly compact set; but then by $(*),\left\{T_{n}\right\}$ is not Cauchy with respect to uniform convergence on this set, a contradiction. Thus Lemma 4.1 is once again applicable.

For each $\varepsilon>0$ and countable, weakly compact subset $K$ of the unit ball of $X$, write

$$
W(K, \varepsilon)=\{T \in A:\|T x\| \leq \varepsilon \text { for all } x \in K\} ;
$$

then the family $W(K, \varepsilon)$ is a neighborhood base at 0 . We show that $A$ is not a $Q$-algebra by showing that each $W(K, E)$ contains an operator which is not quasi-regular in $A$. Since $X$ is nonseparable, there is 
some nonzero $f \in X^{*}$ such that $f(K)=0$. Choose $y \in X$ such that $f(y)=-l$, and let $T=y \otimes f(T x=f(x) y)$. Clearly $T \in W(K, \varepsilon)$. Denoting the identity operator on $X$ by $I$, we have $(T+I) y=0$, so $T+I$ is not invertible in $B(X)$, which implies that $T$ is not quasiregular in $B(X)$, hence neither in $A$.

REMARKS 1. That $A$ is not locally multiplicatively-convex is a consequence of Theorem 2 of Williamson [9]. Indeed, it follows by that theorem that multiplication in $A$ cannot be jointly continuous unless $A$ is normable; however it is easy to see that $A$ is not normable, and in fact it can readily be checked that none of the three examples is metrizable.

2. By Theorem 10 of [3], p. 118, every multiplicative linear functional on $A$ vanishes on the finite-rank operators. Also, if $X$ is a Hilbert space and $A=B(X)$, then $A$ has no nonzero multiplicative linear functionals ([3], p. 115). In general, however, if $A$ is a unital commutative extension of the ideal of compact operators, then $A$ has nonzero multiplicative linear functionals; for example, let $A$ be the algebra generated by the compact operators with the identity operator adjoined.

3. The condition that $A$ be uniformly closed in $B(X)$ is necessary even for $A$ to be pseudocomplete. For if $B_{1}$ is the closure in the topology of $A$ of the set $\{T \in A:\|T\| \leq 1\}$, then $B_{1}$ is a closed, convex, balanced, bounded semigroup in $A$, and the induced norm $\|\cdot\|_{B_{1}}$ on A clearly satisfies $\|\cdot\|_{B_{\perp}} \leq\|\cdot\|$. Theorem 8 of Bonsall [2], p. 161, then implies that $\|\cdot\|_{B_{I}}$ is precisely the operator norm on $A$, from which it follows that $A$ is pseudocomplete only if $A$ is uniformly closed in $B(X)$.

\section{References}

[1] G.R. Allan, "A spectral theory for locally convex algebras", Proc. London Math. Soc. (3) 15 (1965), 399-421. 
[2] F.F. Bonsall, "A minimal property of the norm in some Banach algebras", J. London Math. Soc. 29 (1954), 156-164.

[3] F.F. Bonsall and J. Duncan, Nromerical ranges of operators on normed spaces and of elements of normed algebras (London Mathematical Society Lecture Note Series, 2. Cambridge University Press, Cambridge, 1971).

[4] Nelson Dunford and Jacob T. Schwartz, Linear operators. Part I: General theory (Pure and Applied Mathematics, 7. Interscience, New York, London, 1958).

[5] Taqdir Husain and Shu-Bun $\mathrm{Ng}$, "On the boundedness of multiplicative and positive functionals", J. Austral. Math. Soc. Ser. A 21 (1976), 498-503.

[6] John L. Kelley, General topology (Van Nostrand, Toronto, New York, London, 1955. Reprinted: Graduate Texts in Mathematics, 27. Springer-Verlag, New York, Heidelberg, Berlin, ND [1975]).

[7] Ernst A. Michael, Locally multiplicatively-convex topological algebras (Memoirs of the American Mathematical Society, 11. Amer. Math. Soc., Providence, Rhode Island, 1952).

[8] I.E. Segal, "The group algebra of a locally compact group", Trans. Amer. Math. Soc. 61 (1947), 69-105.

[9] J.H. Williamson, "Two conditions equivalent to normability", J. London Math. Soc. 31 (1956), 111-113.

Department of Mathematics, University of Washington, Seattle, Wash ington, USA. 